\title{
Response and Recovery Times of Elastic and Viscoelastic Capsules in Shear Flow
}

\author{
John Gounley and Yan Peng* \\ Deparment of Mathematics and Statistics, Old Dominion University, Norfolk, \\ VA 23507, USA.
}

Received XXX; Accepted (in revised version) XXX

\begin{abstract}
Amid the recent interest in the role of membrane viscosity in the deformation of a fluid-filled capsule, we consider the role of various capsule properties (shear elasticity, membrane bending stiffness and viscosity) in determining the response and recovery times of a spherical capsule in shear flow. These times are determined by fitting exponential functions to results for the Taylor deformation parameter $D_{x y}$. We focus on the relationship between the membrane and fluid viscosity ratios, as suggested by Diaz et al [8], and whether adjustments to the fluid viscosity ratio may be used to approximate the effects of membrane viscosity. Based on its ability to reproduce response and recovery times, our results suggest that such an approach holds promise.
\end{abstract}

AMS subject classifications: to be provided

Key words: to be provided

\section{Intro}

Diaz and coworkers [9] considered the response and recovery of an elastic capsule in elongational flow. Finding that the capsule's response and recovery times could be determined by an exponential fitting, they investigated the role of parameters such as the fluid viscosity ratio and the capillary number. The response and recovery times of the capsule are important to understanding how a capsule will react in more complicated, time-dependent flows, as arise in medical and industrial applications [10]. Diaz et al [8] extended their consideration to a viscoelastic capsule in elongational flow and compared the respective impacts of different fluid and membrane viscosity ratios.

However, many of the attractive aspects of elongational flow, such as simpler computation due to axisymmetry, may also potentially restrict the applicability of its results. For instance, fluid and membrane viscosity ratios do not affect steady-state shape of the

*Corresponding author. Email addresses: jgounley@odu.edu (J. Gounley), ypeng@odu .edu (Y. Peng) 
capsule in elongational flow, and the capsule's membrane does not exhibit any tanktreading [2]. In contrast, the deformation of a capsule in shear flow has dynamical and angular aspects which do not occur in elongational flow. The steady-state shape of a capsule in shear flow depends on a range of parameters - capillary number, bending stiffness, membrane and fluid viscosity ratios - as do the capsule's angle of inclination and tank-treading frequency.

Nonetheless, Diaz et al [9] applied their exponential-fitting methodology to the results of Ramanujan and Pozrikidis [23] for the deformation of a spherical capsule in shear flow. They found instructive parallels between their results, including a near constant ratio between response times in elongational and shear flows, for capsules with equal steady-state deformation, as measured by the Taylor deformation parameter. This striking comparison, however, is limited because Ramanujan and Pozrikidis did not model the membrane viscosity, and neither study incorporated bending stiffness. We aim to consider this analogy in a more complete setting, with viscoelastic capsules that may resist bending and have a non-unity fluid viscosity ratio.

We consider the deformation response of a spherical capsule in shear flow, along with its shape recovery after the shear flow is abruptly stopped. Our methodology uses a finite element structural method and treats the fluid with a lattice Boltzmann method, coupling the structure and fluid with the immersed boundary method. The structural model considers the capsule's elasticity, membrane viscosity, and bending stiffness, while the fluid model permits different fluid viscosities inside and outside of the capsule. We quantify the shape change of the capsule using the Taylor deformation parameter and fit an exponential curve to this parameter to determine the response and recovery times.

\section{Algorithms}

\subsection{Fluid}

The incompressible Navier-Stokes equations are solved using a lattice Boltzmann method (LBM). Derived from the Boltzmann equation of statistical mechanics, the lattice Boltzmann method considers the fluid to be sets of particles that move between lattice nodes in discrete timesteps with discrete velocities. Despite its statistical origins, the lattice Boltzmann method is deterministic, using the averaged behaviour of particles.

The expression $\mathbf{f}_{i}\left(\mathbf{x}_{j}, t_{n}\right)$ represents the distribution of particles at $\mathbf{x}_{j}$ with velocity $\mathbf{c}_{i}$ at time $t_{n}$. The discrete velocities $\mathbf{c}$ are from the D3Q19 lattice model and we set $h=d x=d t$. Using a multiple relaxation time (MRT) approximation of the collision integral, we have the lattice Boltzmann equation

$$
\mathbf{f}\left(\mathbf{x}_{j}+\mathbf{c} d t, t_{n}+d t\right)-\mathbf{f}\left(\mathbf{x}_{j}, t_{n}\right)=-\mathbf{M}^{-1} \mathbf{S}\left[\mathbf{m}\left(\mathbf{x}_{j}, t_{n}\right)-\mathbf{m}^{(\mathrm{eq})}\left(\mathbf{x}_{j}, t_{n}\right)\right]
$$

in which we denote the probability distribution functions by $\mathbf{f}$, their velocity moments by $\mathbf{m}$, and their equilibrium moments by $\mathbf{m}^{(e q)}[6,7,13]$. 
The lattice Boltzmann method's time evolution thus consists of two steps: collision and streaming. With the multiple relaxation time approximation of the collision kernel, as represented on the right side of Eq. (2.1), probabilities $f_{i}$ at each node $x_{j}$ are mapped to their moment space by the matrix $\mathbf{M}$. Within this moment space, the non-conserved moments $m_{i}\left(\mathbf{x}_{j}, t_{n}\right)$ relax toward their equilibria $m_{i}^{(e q)}\left(\mathbf{x}_{j}, t_{n}\right)$ according to their specified relaxation rates $s_{i}$ on the diagonal of matrix $\mathbf{S}[7,13]$. A final component of the collision term involves the incorporation of the spatial dependent body forces produced by the capsule. We follow the approach of Lallemand et al in updating the flow momentum according to these forces during the collision process, within the moment space $[14,19]$. In the collision phase of LBM, after computing the hydrodynamic moments, momenta $\mathbf{j}=\left[\begin{array}{lll}j_{x} & j_{y} & j_{z}\end{array}\right]$ at gridpoint $\mathbf{x}_{j}$ are updated to according to the forces $\mathbf{p}$, as

$$
\mathbf{j}^{\prime}\left(\mathbf{x}_{j}\right)=\mathbf{j}\left(\mathbf{x}_{j}\right)+\frac{d t}{2} \mathbf{p}\left(\mathbf{x}_{j}\right) .
$$

After using $\mathbf{j}^{\prime}$ to find the equilibrium moments $\mathbf{m}^{(e q)}$, the momentum is updated again, as

$$
\mathbf{j}^{\prime \prime}\left(\mathbf{x}_{j}\right)=\mathbf{j}^{\prime}\left(\mathbf{x}_{j}\right)+\frac{d t}{2} \mathbf{p}\left(\mathbf{x}_{j}\right)
$$

and $\mathbf{j}^{\prime \prime}$ is used to compute the probability distribution after the collision phase.

After the relaxation process is completed, the moments are mapped by matrix $\mathbf{M}^{-1}$ back to the probability distribution functions they represent. Subsequently, in the streaming step represented by the left side of Eq. (2.1), these probabilities $f_{i}\left(x_{j}, t_{n}\right)$ are advected to the next lattice node, $x_{j}+\mathbf{c}_{i} d t$, according to their velocity.

\subsection{Fluid-structure interaction}

The interaction between the capsule and fluid is handled by the immersed boundary method (IBM). IBM due to Peskin $[20,21]$ is a simple method to treat boundary conditions in bio-fluid systems. It has undergone various modifications and improvements to become a useful method for problems of fluid-structure interactions [17]. In IBM, the boundary condition is re-casted as forces due to boundary conditions constraints. IBM maintains two separate grids: an Eulerian fluid grid and a Lagrangian capsule mesh. The set of Lagrangian points is completely independent of the Eulerian mesh, and the interaction between the two grids are realised through a smoothed approximation to the Dirac delta function. We define a 3D version of the discrete delta function as

$$
\begin{aligned}
& \delta_{h}(\mathbf{x})=\delta_{h}\left(x_{1}\right) \delta_{h}\left(x_{2}\right) \delta_{h}\left(x_{3}\right), \quad \mathbf{x} \in R^{3}, \\
& \delta_{h}(x)=\left\{\begin{array}{ll}
\frac{1}{4 h}\left[1+\cos \left(\frac{\pi x}{2 h}\right)\right] & |x| \leq 2 h \\
0 & |x|>2 h
\end{array}\right. \text { ， }
\end{aligned}
$$

which is used to transfer data between the two grids. This is done in two ways: first, local fluid velocities are used to determine the velocity of each mesh node on the capsule 
and, accordingly, update the node's position. The capsule velocity $\mathbf{U}$ at Lagrangian node $\mathbf{X}_{c}$ is determined by

$$
\mathbf{U}\left(\mathbf{X}_{c}\right)=\sum_{j} \delta\left(\mathbf{X}_{c}-\mathbf{x}_{j}\right) \mathbf{u}\left(\mathbf{x}_{j}\right) h^{3}
$$

in which $\mathbf{x}_{j}$ and $\mathbf{u}$ denote Eulerian fluid node position and velocity, respectively. Subsequently, this velocity is used to update the position of the Lagrangian grid by the forward Euler method:

$$
\mathbf{X}_{c}\left(t_{n}+d t\right)=\mathbf{X}_{c}\left(t_{n}\right)+\mathbf{U}\left(\mathbf{X}_{c}\right) d t .
$$

Second, the body forces created by the deformation of the capsule $\mathbf{P}$ at $\mathbf{X}_{c}$ are spread to the nearby fluid nodes by

$$
\mathbf{p}\left(\mathbf{x}_{j}\right)=\sum_{c} \delta\left(\mathbf{X}_{\mathbf{c}}-\mathbf{x}_{j}\right) \mathbf{P}\left(\mathbf{X}_{c}\right),
$$

as the area of the elements has already been incorporated into calculation of $\mathbf{P}$ at $\mathbf{X}_{c}$.

\subsection{Fluid viscosity ratio}

We smooth the viscosity jump across the interface of the capsule, from ambient viscosity $\mu_{a}$ outside of the capsule to internal viscosity $\mu_{c}$, by using the method of Zhang et al [29]. We approximate the shortest normal distance $d$ from a given fluid node to the interface of the capsule. The sign of $d$ is positive or negative if the node is outside or inside of the capsule, respectively. Subsequently, we introduce a Heaviside function of $d$ from [29],

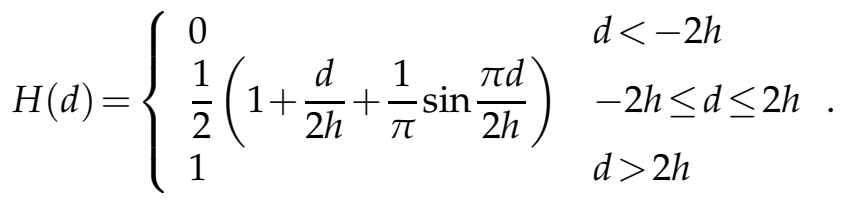

The fluid viscosity $\mu$ at the node is given in terms of $H(d)$ by the equation

$$
\mu(\mathbf{x})=\mu_{c}+\left(\mu_{a}-\mu_{c}\right) H[d(\mathbf{x})] .
$$

\subsection{Viscoelasticity}

In the Kelvin-Voigt model, membrane stress is computed as the sum of elastic and viscous stress resultants; schematically, this may be represented by a spring and dashpot in parallel (see Fig. 1a) [1]. The standard linear solid model, on the other hand, considers a Maxwell element (which has a spring and dashpot in sequence) in parallel with a spring, as in Fig. 1b [1]. Consequently, SLS approximates Kelvin-Voigt as the spring in the Maxwell element becomes sufficiently stiff [27]. This approach has the advantage of combining the improved stability of the standard linear solid model with the timeindependent elastic modulus of Kelvin-Voigt. 


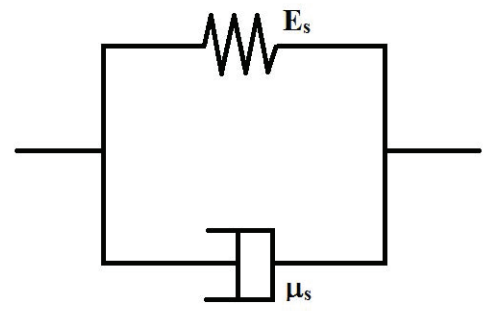

(a)

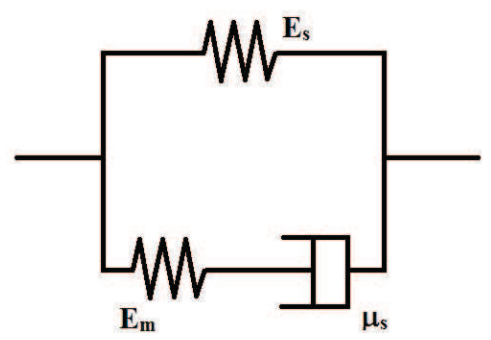

(b)

Figure 1: Schematic of mechanical systems to model membrane viscoelasticity, (a) Kelvin-Voigt and (b) standard linear solid models.

The capsule itself is assumed to be a two-dimensional continuum, composed of triangular elements. Using the approach of Charrier et al [3], undeformed and deformed triangles are mapped to a common plane, in which nodal displacement and deformation gradient matrix $\mathbf{F}$ may be calculated. We employ the non-linear theory of viscoelasticity, which describes the standard linear solid model, with neo-Hookean elasticity. The resulting constitutive stress-strain relation is

$$
\sigma_{i j}=-p \delta_{i j}+g_{0} F_{i k} F_{j k}+\frac{1}{2} F_{i k} F_{j l} \int_{0}^{t} g_{1}(t-\tau) \frac{\partial G_{k l}}{\partial \tau} d \tau
$$

for right Cauchy-Green tensor $\mathbf{G}=\mathbf{F}^{T} \mathbf{F}, g_{0}=E_{s} / 3$ in terms of shear modulus $E_{s}$, and relaxation function $g_{1}(t)=(2 / 3) E_{m} e^{-t / \tau_{1}}[4,25]$. Here, $E_{m}$ is the shear modulus of the Maxwell element and relaxation time constant $\tau_{1}=E_{m} / \mu_{s}$ is given in terms of membrane viscosity $\mu_{s}$. Since we employ a 2D membrane, incompressibility requires that the hydrostatic pressure $p$ is

$$
p=g_{0}|\mathbf{F}|^{-2}+\frac{1}{2} \int_{0}^{t} g_{1}(t-\tau) \frac{\partial|\mathbf{F}|^{-2}}{\partial \tau} d \tau .
$$

Making the reasonable assumption that $\mathrm{G}$ and $|\mathbf{F}|^{-2}$ vary linearly over a sufficiently small timestep, a recurrence relation may be used to drastically simplify the calculation of these integrals [25]. Based on this assumption, we define

$$
I\left(t_{n}\right)=\int_{0}^{t_{n}} e^{-\left(t_{n}-\tau\right) / \tau_{1}} \frac{\partial \mathbf{G}}{\partial \tau} d \tau .
$$

Knowing this integral at time $t_{n}$, we may simplify $I\left(t_{n+1}\right)$ as

$$
\begin{aligned}
I\left(t_{n+1}\right) & =\int_{0}^{t_{n+1}} e^{-\left(t_{n+1}-\tau\right) / \tau_{1}} \frac{\partial \mathbf{G}}{\partial \tau} d \tau \\
& =\int_{0}^{t_{n}} e^{-\left(t_{n+1}-\tau\right) / \tau_{1}} \frac{\partial \mathbf{G}}{\partial \tau} d \tau+\int_{t_{n}}^{t_{n+1}} e^{-\left(t_{n+1}-\tau\right) / \tau_{1}} \frac{\partial \mathbf{G}}{\partial \tau} d \tau
\end{aligned}
$$


and

$$
I\left(t_{n+1}\right)=e^{-d t / \tau_{1}} I\left(t_{n}\right)+\tau_{1} \frac{G\left(t_{n+1}\right)-G\left(t_{n}\right)}{d t}\left(1-e^{-d t / \tau_{1}}\right) .
$$

The same procedure is followed for the integral involving $|\mathbf{F}|^{-2}$ in Eq. (2.12). As a result, the inclusion of membrane viscosity imposes a fairly negligible increase in computational effort, versus a purely elastic model.

A complete explanation and derivation of how forces are calculated is given by Shrivastava and Tang [25], but we briefly outline the procedure here. The load on each triangle is expressed as loads at the triangle's vertices. For each vertex $i$ of a triangle, displacement is expressed in terms of a linear shape functions $N_{i}(x, y)=a_{i} x+b_{i} y+c_{i}$ in the common plane, where the coefficients may be determined from comparing the deformed and undeformed triangles. Then, using the stress $\sigma$ and deformation gradient matrix F for that triangle, the principal of virtual displacements simplies to an expression for forces

$$
\left[\begin{array}{l}
P_{x}(i) \\
P_{y}(i)
\end{array}\right]=\sigma \mathbf{F}^{-T} \nabla N_{i} V_{v o l}
$$

Here, $P_{x}(i)$ and $P_{y}(i)$ represent the forces in the $\mathrm{x}$ - and y-directions of the common plane at node $i$, and $V_{v o l}$ is the element volume. These two-dimensional forces are then mapped back to the global coordinates and summed over every triangle.

\subsection{Bending}

Helfrich's formulation of the bending energy of a zero-thickness membrane is given as

$$
W_{b}=\frac{E_{B}}{2} \int_{s}\left(2 \kappa-c_{0}\right)^{2} d S
$$

for bending stiffness modulus $E_{B}$, capsule surface area $S$, mean curvature $\kappa$, and spontaneous curvature $c_{0}$ [18]. From this, Helfrich derives the bending force density at a node as

$$
\mathbf{f}_{b}=E_{B}\left[\left(2 \kappa+c_{0}\right)\left(2 \kappa^{2}-2 \kappa_{g}-c_{0} \kappa\right)+2 \Delta_{L B} \kappa\right] \hat{\mathbf{n}},
$$

in which $\kappa_{g}$ is the Gaussian curvature [28]. The curvature of the capsule at each node was calculated using quadratic surface fitting [11]. The discrete Laplace-Beltrami operator, acting on mean curvature $\kappa$ at node $\mathbf{p}$, may be represented as

$$
\Delta_{L B} \kappa(\mathbf{p})=\frac{1}{d} \sum_{j \in N(\mathbf{p})} \omega_{j}\left[\kappa(\mathbf{p})-\kappa\left(\mathbf{x}_{j}\right)\right]
$$

for nodes $\mathbf{x}_{j}$ belonging to the set $N(\mathbf{p})$ of nodes which neighbor $\mathbf{p}$ [24], as shown in Fig. 2 . We follow Pinkall and Polthier [22] in setting the weights $\omega_{j}$ as

$$
\omega_{j}=\frac{\cot \left(\alpha_{j}\right)+\cot \left(\beta_{j}\right)}{2}
$$




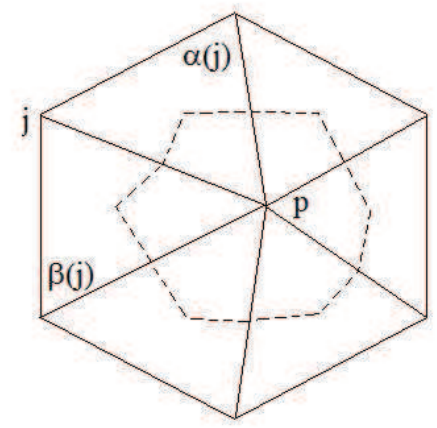

Figure 2: Neighborhood of vertex point $P$ on the spherical surface.

in which $\alpha_{j}$ and $\beta_{j}$ are defined as in Fig. 2. Similarly, we define normalization factor $d$ with the Voronoi region $A_{\text {vor }}$ (enclosed by the dotted line in Fig. 2),

$$
d=A_{\text {vor }}=\frac{1}{8} \sum_{j \in N(\mathbf{p})}\left[\cot \left(\alpha_{j}\right)+\cot \left(\beta_{j}\right)\right]\left\|\mathbf{p}-\mathbf{x}_{j}\right\|^{2}
$$

as given by [11].

\section{Numerical performance}

We consider a single spherical capsule in simple shear flow, looking at both an underformed capsule being deformed by the shear flow and a deformed capsule recovering after shear flow has been stopped. The simulation domain is defined in terms of the equivalent radius $a$ of the capsule, as $[0,10 a] \times[0,10 a] \times[0,8 a]$. In this domain, the flow direction is parallel to the $x$-axis and the direction of the velocity gradient is along the $y$-axis. Li and Sarkar [16] have shown that a domain of this size is sufficient to avoid wall effects in shear flow. The time step $d t$ and spatial step $d x$ are identical: $h=d x=d t=0.08 a$. The simple shear flow, in terms of shear rate $k$, is

$$
\mathbf{u}=\left[\left(y-\frac{1}{2} H\right) k, 0,0\right]
$$

in which $H=10 a$, the $y$-direction size of the domain. The Reynolds number is $\operatorname{Re}=$ $\rho a^{2} k / \mu_{a}=0.05$ and hence, the effect of inertia is negligible. The initial center of capsule is placed in the center of the domain, with respect to $x-, y-$, and $z$-axes. The deformation of the capsule is quantified in terms of the Taylor deformation parameter $D(t)=(L-W) /(L+W)$, with $L$ and $W$ representing the major and minor axes of an ellipsoid in the $x y$-plane with the same moment of inertia as the viscoelastic capsule [5].

Several non-dimensional parameters govern our simulations. The capillary number (or, equivalently, the non-dimensional shear rate) is $C a=\mu_{a} k a / E_{s}$, in which $k$ is the dimensional shear rate, $\mu_{a}$ is the ambient fluid viscosity, $a$ is the radius of the capsule, and $E_{s}$ is 


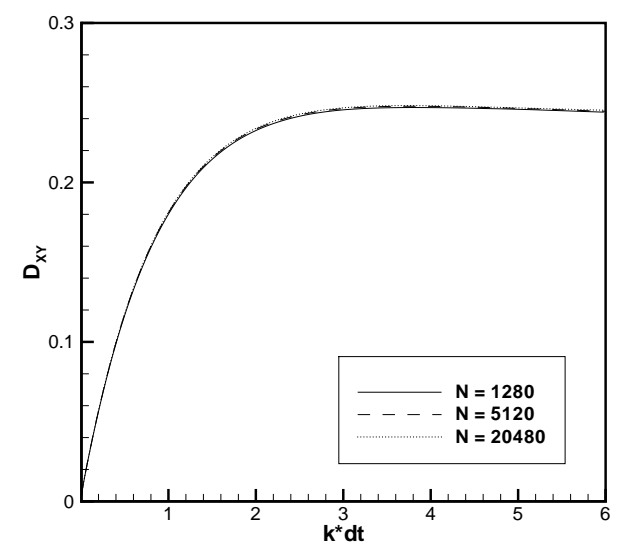

(a)

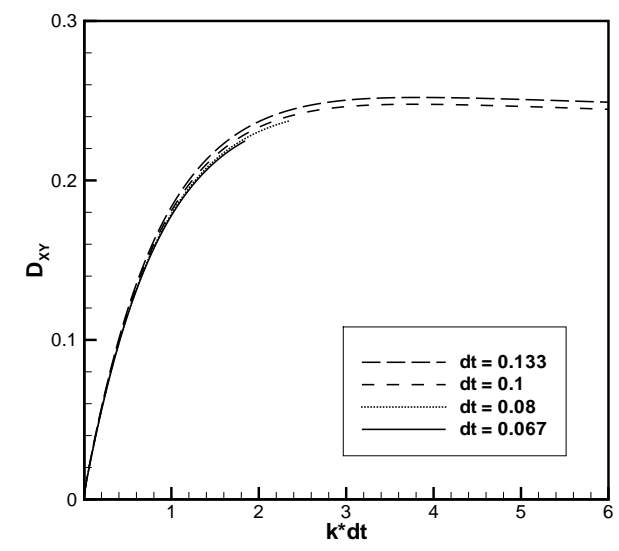

(b)

Figure 3: (a) Convergence analysis of SLS model with meshes composed of $N=1280,5120$, and 20480 triangles, with parameters $C a=0.05, \mu_{S}=10 \mu_{a}$, and $G=E_{m} / E_{S}=50$. (b) Convergence analysis of SLS model with Eulerian grid $d x=d t=0.133,0.1,0.08$ and 0.067 , with parameters $C a=0.05, \mu_{s}=10 \mu_{a}$, and $G=E_{m} / E_{s}=50$.

the shear elasticity modulus. The bending stiffness ratio is $E_{b}=E_{B} /\left(a^{2} E_{s}\right)$, for bending stiffness modulus $E_{B}$. The fluid viscosity ratio is $V=\mu_{c} / \mu_{a}$ and the membrane viscosity ratio is $\eta=\mu_{s} /\left(a \mu_{c}\right)$, defined in terms of the fluid viscosity $\mu_{c}$ inside the capsule and the membrane viscosity coefficient $\mu_{s}$. We also consider deformation and recovery with respect to several pertinent timescales, using the notation of [27]. The elastic timescale is $\tau_{e}=V \mu_{a} a / E_{s}$ and, for capsules with bending stiffness, the timescale associated with bending is $\tau_{b}=V \mu_{a} a^{3} / E_{B}$.

The convergence of this computational framework with respect to the Lagrangian and Eulerian grids is considered in Fig. 3. Varying the number of triangles $N$ composing the capsule shows that the method converges nicely with respect to the Lagrangian mesh. Likewise, with a constant $N=5120$, the method is also observed to converge with respect to the Eulerian grid. These results are consistent with comparable, but simpler frameworks, in which similar convergence was observed (e.g., [26]). In light of these results, subsequent simulations employ grids with $N=5120$ and $d x=d t=0.08$.

We compared the method's accuracy with existing methods for deformation of 3D spherical capsules in shear flow. Regarding elasticity, our results agreed well with an existing lattice Boltzmann method from Sui et al [26] and the boundary integral method of Ramanujan and Pozrikidis [23], for the different values of capillary number Ca shown in Fig. 4a. All three methods compare well for these purely elastic capsules. Another simple comparison, with the thin-shelled method of [15], was conducted for bending stiffness; the bending modulus is varied in Fig. $4 \mathrm{~b}$. A slight difference is noted for $E_{b}=0$, but the results agree well otherwise. Results for a fluid viscosity ratio of $V=5$ are shown 
in Fig. 4c, along with those of [26] and the boundary integral method of [23], for different capillary numbers. Our results compare reasonably well with those from the more accurate boundary integral method; while there is some initial overshooting, the steadystate results approach more closely [23] than Sui et al. Finally, our SLS implementation is compared with that of Yazdani and Bagchi in Fig. 4d. This comparison is necessarily inexact, as a neo-Hookean shear elasicity is used here, while Yazdani and Bagchi used a Skalak constitutive law with $C=1$. Nonetheless, the comparision and convergence as the ratio $G=E_{m} / E_{s}$ becomes large is clear. In all subsequent results, $G=50$ is used in SLS to approximate Kelvin-Voigt.

\section{Results}

We compared the response and recovery times of elastic or viscoelastic capsules in shear flow. We determine the response time $\tau_{s}$ in terms of the fluid shear rate $k$ with the model

$$
D_{x y}(t)=D_{\max }\left[1-e^{-t /\left(k \tau_{s}\right)}\right]
$$

If $D_{x y}(t)$ has a near-constant equilibrium value after deformation, $D_{\max }$ is this value; otherwise, $D_{\max }$ is defined as the first local maximum of $D_{x y}(t)$. Conversely, the model

$$
D_{x y}(t)=D_{\text {stop }}\left[e^{-t /\left(k \tau_{r}\right)}\right]
$$

defines the recovery time $\tau_{r}$ in terms of $k$, and the value of $D_{\text {stop }}$ is the value of $D_{x y}(t)$ when shear flow is stopped. Since $D_{\max }$ and $D_{\text {stop }}$ are factored out, the response and recovery times characterize how quickly the capsule arrives at the steady-state deformation and recovers the original shape, respectively, independent on the extent of deformation or recovery. A large response or recovery time indicates a slower response or recovery process, since it describes a slow exponential decay in the models.

The values $\tau_{s}$ and $\tau_{r}$ are determined by fitting these exponential functions to our simulation results, using a least squares method. In general, exponential fittings had a correlation coefficient $R^{2}>0.99$. For two kinds of capsules, however, this failed to be the case. First, the response model had only correlation coefficients $R^{2}>0.97$ for large $V$ and $\eta$. Second, the recovery model was much poorer for very large bending stiffness, with $R^{2}$ as low as 0.92. In contrast, Diaz et al's fitting for response times in elongational flow had correlation coefficients in excess of 0.98 [8].

\subsection{Elasticity and bending stiffness}

We first consider the separate roles of the capsule's elasticity and bending stiffness. For elasticity, we consider various capillary numbers and normalize response and recovery times by elastic timescale $\tau_{e}$. If we consider, in Fig. $5 \mathrm{a}$, a capsule with $E_{b}=0.025, \eta=0$, and $V=1$, we see that $\tau_{r} / \tau_{e} \approx \tau_{s} / \tau_{e}$ for small $C a$, but they diverge as $C a$ increases. While $\tau_{r} / \tau_{e}$ 


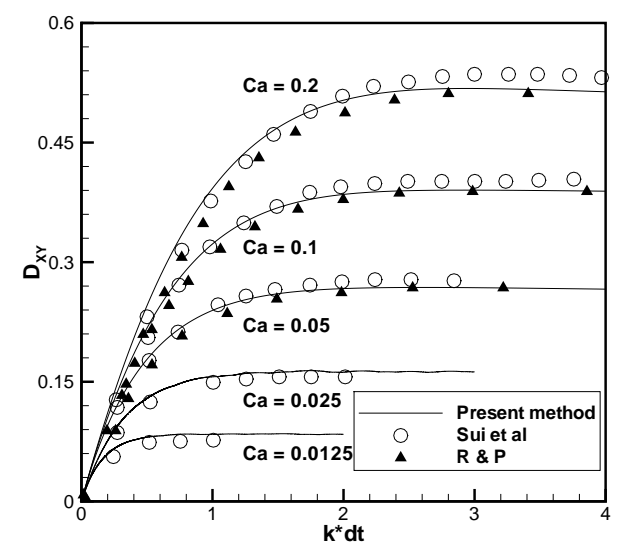

(a)

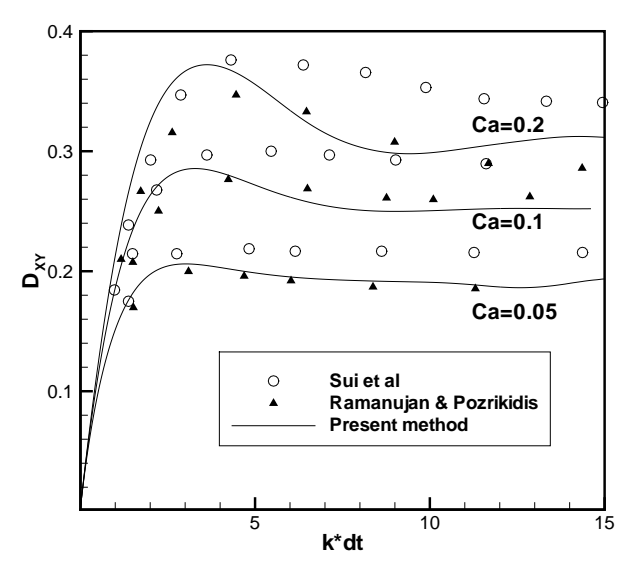

(c)

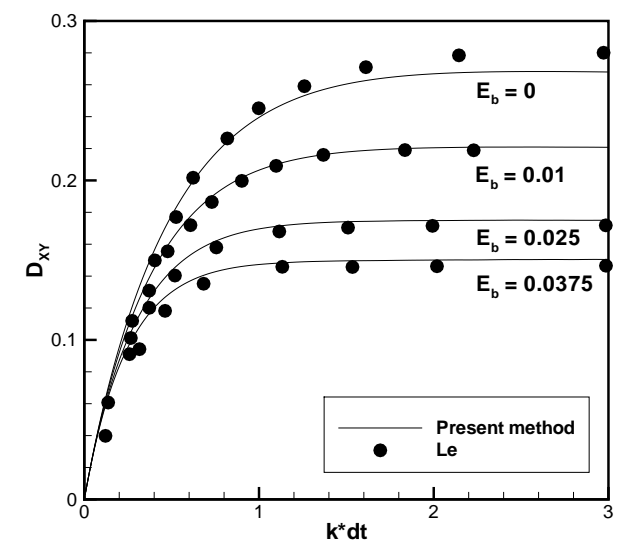

(b)

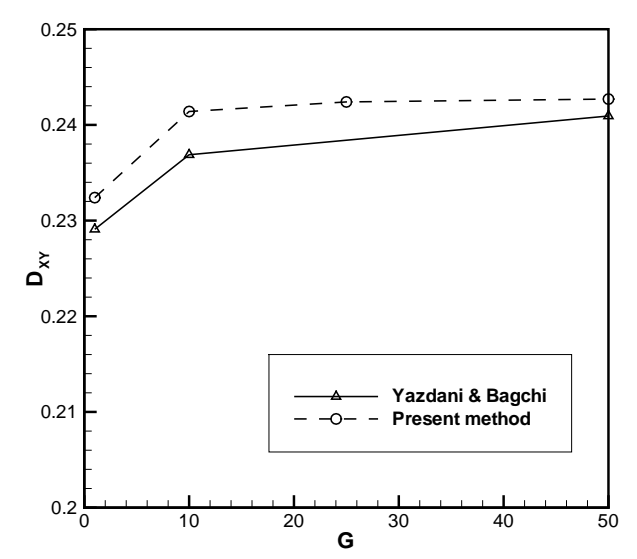

(d)

Figure 4: Comparisons with Sui et al (open circles), Ramanujan and Pozrikidis (filled triangles), Le (filled circles), and Yazdani and Bagchi (open triangles). (a) Varied $C a$ for $E_{b}=0, V=1$, and $\eta=0$. (b) Varied $E_{b}$ for $C a=0.05, V=1$, and $\eta=0$. (c) For $V=5$, varied $C a$ with $E_{b}=0$ and $\eta=0$. (d) Varied $G=E_{m} / E_{s}$ for $C a=0.1$, $E_{b}=0, V=1$, and $\eta=10$.

stays nearly constant, $\tau_{s} / \tau_{e}$ continues to decrease. As [9] suggests, this contrast seems reasonable, since recovery is driven by the stored energy in the capsule and the deformation is caused by a continual outside force. However, Diaz et al find that $\tau_{s} / \tau_{e}$ reaches a minimum value near $C a=0.05$ and rises for larger capillary numbers. This difference, however, perhaps may be attributed to the differences between what constitutes a 'large' capillary number in elongational and shear flows. Thus, while we do not observe an increase for $C a \leq 0.4$, this may still occur at higher $C a$. 


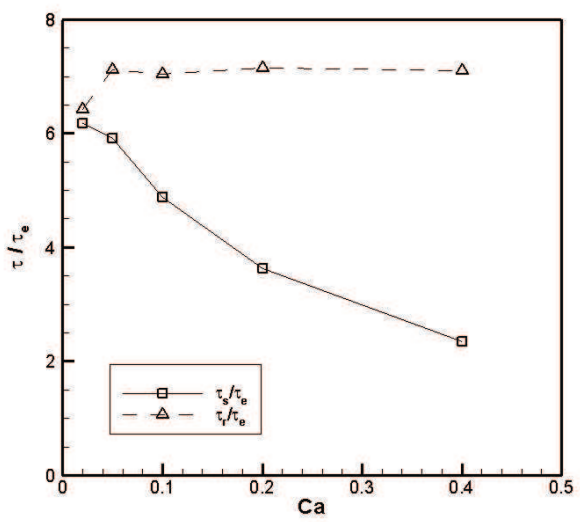

(a) Normalized response $\tau_{s} / \tau_{e}$ (solid lines) and recovery $\tau_{r} / \tau_{e}$ (dotted lines) are plotted with respect to $\mathrm{Ca}$.

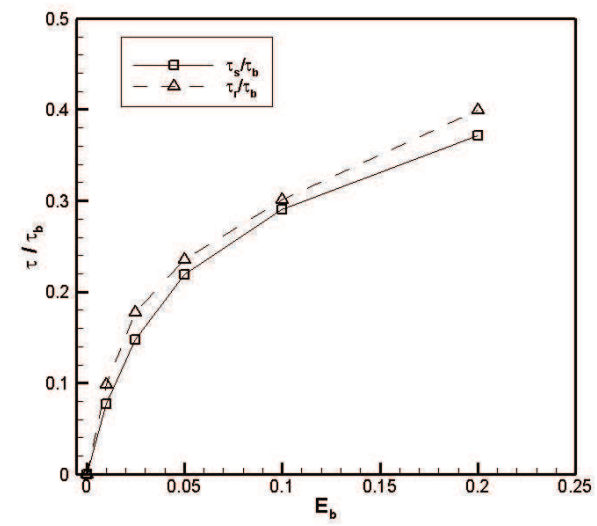

(b) Normalized response $\tau_{s} / \tau_{b}$ (solid lines) and recovery $\tau_{r} / \tau_{b}$ (dotted lines) are plotted with respect to $E_{b}$.

Figure 5: Effect of elasticity and bending stiffness on the normalized response and recovery times. Other parameters in $5 \mathrm{a}$ are $E_{b}=0.025, V=1$, and $\eta=0$. Other parameters in $5 \mathrm{~b}$ are $C a=0.05, V=1$, and $\eta=0$.

A contrasting result is found when varying the bending stiffness and normalizing with respect to the bending stiffness timescale $\tau_{b}$. Fig. $5 \mathrm{~b}$ considers a capsule with $C a=$ $0.05, M=0$, and $V=1$. We find that $\tau_{r} / \tau_{b} \approx \tau_{s} / \tau_{b}$ over the range of bending stiffnesses considered. Since bending stiffness strictly considers how the capsule's shape compares with its preferred shape, and is not directly related to the fluid, it seems to act similarly in response and recovery. Further, the positive inclination suggest that the dependency of characteristic times on bending stiffness decreases as $E_{b}$ increases.

\subsection{Fluid and membrane viscosity ratios}

Recent work has suggested that the effects of the fluid and membrane viscosity ratios in both shear and elongational flows are qualitatively similar [8,27]. Such studies suggest that membrane viscosity might be modeled by artificially altering the fluid viscosity ratio, an idea dating back to Keller and Skalak [12]. The idea is attractive, given the comparative ease with which non-unity fluid viscosity ratios may be implemented in current computational schemes. Two questions seem pertinent here: Are the roles of the fluid and membrane viscosity ratios independent in shear flow? Further, can a simple relationship between their characteristic times be established, as was the case with elongational flow?

The independent roles of fluid and membrane viscosity ratios in determining response times has been noted for elongational flow. Diaz et al found that a bilinear model in $\eta$ and $V$ sufficed to describe $k \tau_{s}$, as

$$
k \tau_{s}=a_{s}(C a) \eta+b_{s}(C a) V+c_{s}(C a)
$$


Table 1: Coefficients from fitting response and recovery times for a range of viscoelastic capsules using Eqs. (4.5) and (4.6). The adjacent ranges are the bounds of a $95 \%$ confidence interval.

\begin{tabular}{|c|c|c|c|}
\hline$k \tau$ & $a\left(C a, E_{b}\right)$ & $b\left(C a, E_{b}\right)$ & $c\left(C a, E_{b}\right)$ \\
\hline$k \tau_{s}\left(C a=0.05, E_{b}=0\right)$ & $0.0208 \pm 0.0039$ & $0.0590 \pm 0.0149$ & $0.4158 \pm 0.0638$ \\
$k \tau_{r}\left(C a=0.05, E_{b}=0\right)$ & $0.0534 \pm 0.0028$ & $0.2711 \pm 0.0109$ & $0.3986 \pm 0.0466$ \\
\hline$k \tau_{s}\left(C a=0.05, E_{b}=0.025\right)$ & $0.0157 \pm 0.0039$ & $0.0550 \pm 0.0158$ & $0.2567 \pm 0.0638$ \\
$k \tau_{r}\left(C a=0.05, E_{b}=0.025\right)$ & $0.0299 \pm 0.0018$ & $0.1380 \pm 0.0075$ & $0.2209 \pm 0.0303$ \\
\hline$k \tau_{s}\left(C a=0.2, E_{b}=0\right)$ & $0.0417 \pm 0.0110$ & $0.1464 \pm 0.0410$ & $0.685 \pm 0.1402$ \\
$k \tau_{r}\left(C a=0.2, E_{b}=0\right)$ & $0.2438 \pm 0.0128$ & $1.0120 \pm 0.0477$ & $1.614 \pm 0.162$ \\
\hline
\end{tabular}

with coefficients $a_{s}, b_{s}, c_{s}$ depending on $C a$ [8]. In particular, for large $C a$, they found $a_{s} \approx b_{s}$. Diaz et al use these coefficients to posit an artificial fluid viscosity ratio,

$$
V^{*}=V+\frac{a_{s}}{b_{s}} \eta
$$

for which an elastic capsule would have the same response time as a viscoelastic capsule. We considered whether a comparable pair of bilinear equations,

$$
\begin{aligned}
k \tau_{s} & =a_{s}\left(C a, E_{b}\right) \eta+b_{s}\left(C a, E_{b}\right) V+c_{s}\left(C a, E_{b}\right), \\
k \tau_{r} & =a_{r}\left(C a, E_{b}\right) \eta+b_{r}\left(C a, E_{b}\right) V+c_{r}\left(C a, E_{b}\right),
\end{aligned}
$$

might fit our data, with the constant coefficients now functions of the capillary number and bending stiffness. A successful fit would show the independence of the fluid and membrane viscosity ratios and suggest possible relationships for shear flow.

We simulated three ensembles of capsules: $C a=0.05$ and $E_{b}=0, C a=0.05$ and $E_{b}=$ 0.025 , and $C a=0.2$ and $E_{b}=0$. Plausible fits for the characteristics of all three ensembles were found, using equations (4.5) and (4.6), and tabulated in Table 1.

The bilinear model was found to fit very well for $k \tau_{r}$, with a correlation coefficient $R^{2}>0.99$ for all three cases. The model fit more poorly for $k \tau_{s}$, with $R^{2} \approx 0.91$ for $\mathrm{Ca}=0.05$ and $E_{b}=0, R^{2} \approx 0.96$ for $\mathrm{Ca}=0.05$ and $E_{b}=0.025$, and $R^{2} \approx 0.95$ for $\mathrm{Ca}=0.2$ and $E_{b}=0$. As a result, the accuracy of the coefficients for $k \tau_{s}$ does not approach that of Diaz et al. Given that the coefficients for $k \tau_{s}$ reflect the error from the exponential model fittings being compounded with the bilinear model fitting, we considered these levels of error to be acceptable. A few instructive inferences are apparent from the data:

The coefficients $a_{s}$ and $b_{s}$ for $k \tau_{s}$ and both ensembles with $\mathrm{Ca}=0.05$ are surprisingly similar, suggesting that their dependence on $E_{b}$ may be small. More interesting, however, are the ratios $b_{s} / a_{s}$ and $b_{r} / a_{r}$. Starting from the base ensemble for $\mathrm{Ca}=0.05$ and $E_{b}=0$ with $b_{s} / a_{s} \approx 2.8$, the ratio of response coefficients increases to $b_{s} / a_{s} \approx 3.5$ with the both addition of bending stiffness $E_{b}=0.025$ and the higher capillary number $\mathrm{Ca}=0.2$. Conversely, the ratio $b_{r} / a_{r} \approx 5$ for the base ensemble decreases to $b_{r} / a_{r} \approx 4.6$ with the inclusion of bending stiffness $E_{b}=0.025$ and falls to $b_{r} / a_{r} \approx 4.1$ at the higher capillary 
number $\mathrm{Ca}=0.2$. These ratios for the response coefficients do not align with Diaz et al, who observed $b_{s} / a_{s} \approx 2$ for $\mathrm{Ca}=0.005$ and $b_{s} / a_{s} \approx 1$ for $\mathrm{Ca}=0.05$ in elongational flow. Thus, while Diaz et al observe a decrease in the ratio $b_{s} / a_{s}$ as Ca becomes larger in elongational flow, a modest increase is observed in shear flow.

We consider whether this model fits sufficiently well to satisfy and extend Diaz et $a l^{\prime}$ s hypothesis by comparing the response and recovery times of our own viscoelasticity model with the artificial viscosity model. This comparison may be presented in two ways. First, the membrane viscosity ratio $\eta$ may be integrated into an artificial fluid viscosity ratio $V^{*}$, as

$$
V^{*}=V+\frac{a}{b} \eta
$$

Second, an artificial fluid viscosity ratio $V^{*}$, in excess of the actual fluid viscosity ratio $V$, may be interpreted as an artificial membrane viscosity, as

$$
\eta^{*}=\frac{b}{a}\left(V^{*}-V\right)
$$

To consider whether these models fit sufficiently well to satisfy and extend Diaz et al's hypothesis, a comparison is made between the response and recovery times of the KelvinVoigt viscoelasticity model and the artificial fluid viscosity ratio model. First, an artificial membrane viscosity ratio $\eta$ is used to simulate the effects of a different fluid viscosity ratio $V^{*}$, as in equation (4.7). In Fig. 6a, square data points depict the characteristic times of capsules with $C a=0.05, E_{b}=0$, and $\eta=0$, plotted against their different fluid viscosity ratios $V$. In contrast, the triangular data points are the characteristic times of capsules with identical $C a$ and $E_{b}$, but with $V=0.2$ and various $\eta$. The $x$-axis values for these triangular data points are $V^{*}$ in equation (4.7), derived by plugging in $V=0.2$ and the particular value of $\eta$. The same methodology is used in Fig. $6 \mathrm{~b}$, in which square data points represent characteristic times for $C a=0.05, E_{b}=0.025$, and $\eta=0$, with various fluid viscosity ratios. On the other hand, the triangular data comes from the characteristic times of capsules with the same $C a$ and $E_{b}$, but $V=1$ and different values of $\eta$. In both cases, the derived relationship between $V$ and $\tau_{r}$ matches very well. The results for $\tau_{s}$ are still acceptable, but noticeable differences emerge. This is unsurprising, given the exponential fittings for $\tau_{s}$, particularly at large $V$ and $\eta$, were much poorer than for $\tau_{r}$.

Second, the same results may also be viewed through the lens of the membrane viscosity ratio. In Figs. $7 \mathrm{a}$ and $7 \mathrm{~b}$, square data points are characteristic times of capsules plotted against their membrane viscosity ratios $\eta$. In Fig. 7a, other parameters are $C a=0.05$, $E_{b}=0$, and $V=0.2$, while the parameters are $C a=0.05, E_{b}=0.025$, and $V=1$ for Fig. $7 \mathrm{~b}$. Triangular data points, on the other hand, originate in capsules with the same $\mathrm{Ca}$ and $E_{b}$ values, but have $\eta=0$ and various fluid viscosity ratios $V^{*}$. In these cases, the "extra" fluid viscosity $V^{*}-V$ is used to determine the artificial membrane viscosity $\eta$, using equation (4.8), which provides their $x$-axis values. 


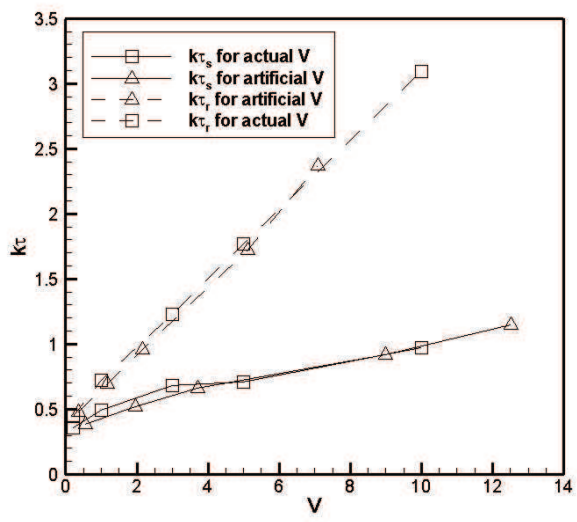

(a)

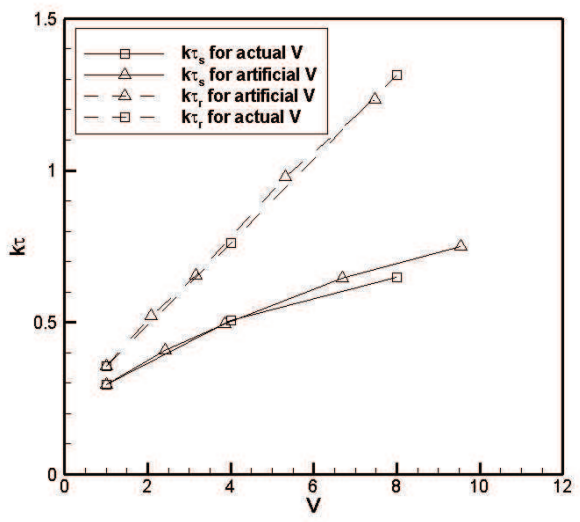

(b)

Figure 6: Characteristic times $k \tau_{s}$ (solid lines) and $k \tau_{r}$ (dotted lines) for actual fluid viscosity ratios (squares) and artificially calculated fluid viscosity ratios (triangles). The capillary number is $C a=0.05 . E_{b}=0$ in $6 a$ and $E_{b}=0.025$ in $6 \mathrm{~b}$.

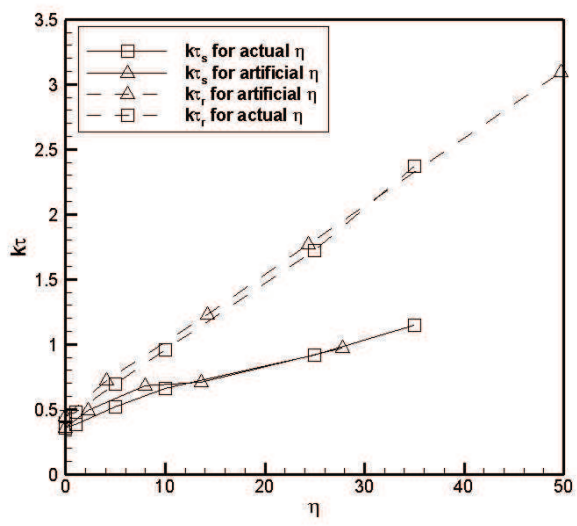

(a)

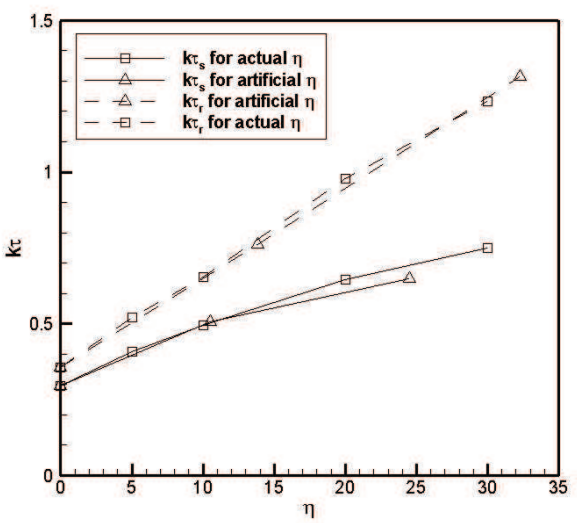

(b)

Figure 7: Characteristic times $k \tau_{s}$ (solid lines) and $k \tau_{r}$ (dotted lines) for actual membrane viscosity ratios (squares) and artificially calculated membrane viscosity ratios (triangles). The capillary number is $C a=0.05$. $E_{b}=0$ in $7 \mathrm{a}$ and $E_{b}=0.025$ in $7 \mathrm{~b}$.

\subsection{Limitations}

Diaz et al note agreement for $\tau_{s}$ between two spherical capsules does not imply that every aspect of their local deformation will be identical [8]. This limitation is even stronger for shear flow, as neither the equilibrium Taylor deformation parameter or angle of inclination will necessarily be the same, even if the response or recovery times match. For 


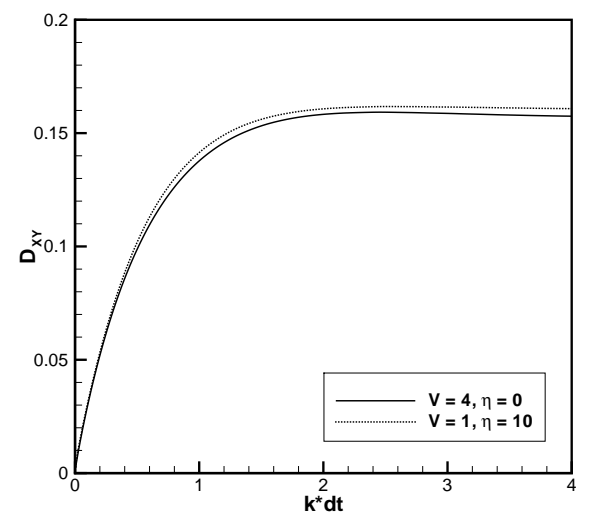

(a)

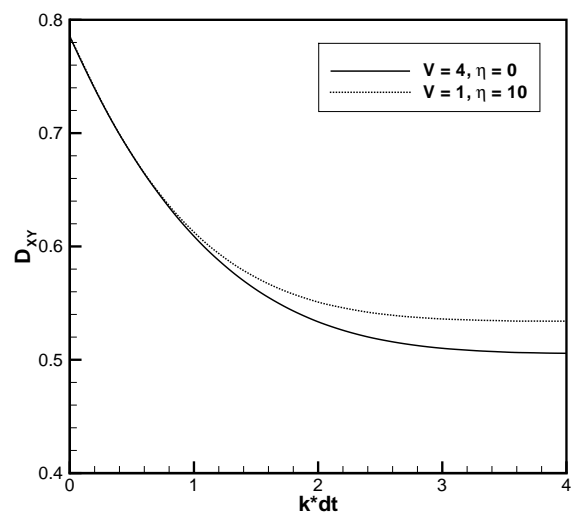

(b)

Figure 8: Comparison of deformation $D_{x y}(\mathrm{a})$ and inclination angle $\theta$ (b) of two capsules with nearly identical response times $\tau_{s}$. The capillary number is $C a=0.05$ and $E_{b}=0.025$.

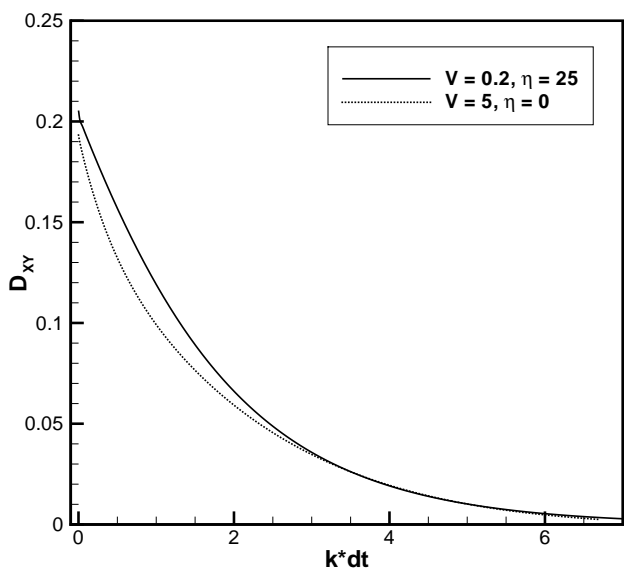

Figure 9: Comparison of $D_{x y}$ during shape recovery of two capsules with nearly identical recovery times $\tau_{r}$. The capillary number is $C a=0.05$ and $E_{b}=0$.

instance, Figs. $8 \mathrm{a}$ and $8 \mathrm{~b}$ consider the deformation and inclination angle, respectively, of two capsules with nearly identical response times $\tau_{s}$. Nonetheless, their steady-state deformation and inclination angles differ. Likewise, Fig. 9 shows the Taylor deformation parameter during shape recovery of two capsules with nearly equal recovery times $\tau_{r}$. Although this latter pair of capsules had similar equilibrium deformations, their recovery courses appear different, despite the qualitative similarity reflected in the recovery times. 
As a result, it is not clear that this sort of approach will lead to a viable computational algorithm, as three prominent limitations emerge. First, significant a priori knowledge of the ratio $b / a$ is necessary to simulate one form of viscosity with the other. Second, the ratios $b / a$ for response and recovery of a single capsule are not, in general, equal. Finally, even if the response and recovery times are accurately modeled, this does not generally imply that the capsule's deformation or inclination angle will be indentical too.

Still, it represents an useful parallel for relating fluid and membrane viscosity ratios. Recently, Yazdani and Bagchi have suggested a comparable model, $V^{*}=V+\phi$, in which $\phi$ is the ratio of membrane dissipation to dissipation inside the capsule. Yazdani and Bagchi show that this approach is effective in recovering a spherical capsule's tank-treading frequency. This result is particularly promising, in that it deals more with capsule dynamics than the capsule shape changes considered here; ideally, such a model for replacing membrane viscosity would reasonably describe both dynamics and shape changes.

\section{Conclusion}

We found that the deformation of a spherical capsule in shear flow, as quantified by the Taylor deformation parameter, cannot be fit by an exponential curve with the same accuracy observed for elongational flow. Still, the results are sufficient to display the contrasting dependence of the response and recovery times on the capillary number, along with their equivalent relation to bending stiffness. Further, for the range of data considered, these characteristic times may be modeled by bilinear functions of the fluid and membrane viscosity ratios $V$ and $\eta$. The fit of these functions is sufficient to show that an artificial viscosity model may be used to recover the approximate characteristic times of a viscoelastic capsule. Alternately, it seems that one could also simulate a fluid viscosity ratio using artificial membrane viscosity, though it is not clear that a need exists for such an approach.

It would be interesting to see whether the approach outlined here may be extended to the deformation and recovery of a biconcave capsule. While more complex geometries are less amenable to the sort of exponential analysis performed here, even a qualitative agreement between the effects of membrane viscosity and an artificial fluid viscosity may be useful.

\section{Acknowledgments}

Y. Peng acknowledges support from Old Dominion University Research Foundation Grant \#503921 and National Science Foundation Grant DMS-1319078. J. Gounley acknowledges support from the Old Dominion University Modeling and Simulation Initiative. 


\section{References}

[1] K. Athanasiou and R. Natoli, Introduction to Continuum Biomechanics, Synthesis Lectures on Biomedical Engineering, Morgan \& Claypool Publishers, 2008.

[2] K. Chang and W. Olbricht, Experimental studies of the deformation and breakup of a synthetic capsule in steady and unsteady simple shear flow, J. Fluid Mech., 250 (1993), pp. 609-633.

[3] J. Charrier, S. Shrivastava, and R. Wu, Free and constrained inflation of elastic membranes in relation to thermoforming - non-axisymmetric problems, J. Strain Anal. Eng. Des., 24 (1989), pp. 5574.

[4] R. Christensen, A nonlinear theory of viscoelasticity for application to elastomers, J. Appl. Mech., 47 (1980), pp. 762-768.

[5] J. R. Clausen and C. K. Aidun, Capsule dynamics and rheology in shear flow: Particle pressure and normal stress, Phys. Fluids, 22 (2010), p. 123302.

[6] D. d'Humières, Generalized lattice Boltzmann equations, Prog. Astronaut. Aeronaut., 159 (1992), pp. 450-458.

[7] D. d'Humières, I. Ginzburg, M. Krafczyk, P. Lallemand, and L.-S. Luo, Multiple-relaxationtime lattice Boltzmann models in three dimensions, Phil. Trans. R. Soc. A, 360 (2002), pp. 437-451.

[8] A. Diaz, D. Barthès-Biesel, and N. Pelekasis, Effect of membrane viscosity on the dynamic response of an axisymmetric capsule, Phys. Fluids, 13 (2001), pp. 3835-3838.

[9] A. Diaz, N. Pelekasis, and D. Barthes-Biesel, Transient response of a capsule subjected to varying flow conditions: Effect of internal fluid viscosity and membrane elasticity, Phys. Fluids, 12 (2000), pp. 948-957.

[10] E. A. Evans, Structure and deformation properties of red blood cells: Concepts and quantitative methods, Meth. Enzymol., 173 (1989), pp. 3-35.

[11] R. V. Garimella and B. K. Swartz, Curvature estimation for unstructured triangulations of surfaces, Tech. Rep. Tech. Rep. LA-UR-03-8240, Los Alamos National Laboratory, 2003.

[12] S. R. Keller and R. Skalak, Motion of a tank-treading ellipsoidal particle in a shear flow, J. Fluid Mech., 120 (1982), pp. 27-47.

[13] P. Lallemand and L.-S. Luo, Theory of the lattice Boltzmann method: Acoustic and thermal properties in two and three dimensions, Phys. Rev. E, 68 (2003), p. 036706.

[14] P. Lallemand, L.-S. Luo, and Y. Peng, A lattice Boltzmann front-tracking method for interface dynamics with surface tension in two dimensions, J. Comput. Phys, 226 (2007), pp. 1367-1384.

[15] D. V. Le, Effect of bending stiffness on the deformation of liquid capsules enclosed by thin shells in shear flow, Phys. Rev. E, 82 (2010), p. 016318.

[16] X. Li and K. Sarkar, Front tracking simulation of deformation and buckling instability of a liquid capsule enclosed by an elastic membrane, J. Comput. Phys, 227 (2008), pp. 4998-5018.

[17] R. Mittal and G. Iaccarino, Immersed boundary methods, Annu. Rev. Fluid Mech., 37 (2005), pp. 239-261.

[18] Z.-C. Ou-Yang and W. Helfrich, Bending energy of vesicle membranes: General expressions for the first, second, and third variation of the shape energy and applications to spheres and cylinders, Phys. Rev. A, 39 (1989), p. 5280.

[19] Y. Peng and L.-S. Luo, A comparative study of immersed-boundary and interpolated bounce-back methods in LBE, Progr. Comput. Fluid Dynam. Int. J., 8 (2008), pp. 156-167.

[20] C. S. Peskin, Numercial analysis of blood flow in the heart, Phys. Fluids, 13 (1997), pp. 3452-3459.

[21] C. S. Peskin, The immersed boundary method, Acta Numer., 11 (2002), pp. 479-517.

[22] U. Pinkall and K. Polthier, Computing discrete minimal surfaces and their conjugates, Exper. Math., 2 (1993), pp. 15-36. 
[23] S. Ramanujan and C. Pozrikidis, Deformation of liquid capsules enclosed by elastic membranes in simple shear flow: Large deformations and the effect of fluid viscosities, J. Fluid Mech., 361 (1998), pp. 117-143.

[24] M. Reuter, S. Biasotti, D. Giorgi, G. Patanè, and M. Spagnuolo, Discrete Laplace-Beltrami operators for shape analysis and segmentation, Comput. Gr., 33 (2009), pp. 381-390.

[25] S. Shrivastava and J. Tang, Large deformation finite element analysis of non-linear viscoelastic membranes with reference to thermoforming, J. Strain Anal. Eng. Des., 28 (1993), pp. 31-51.

[26] Y. Sui, H. Low, Y. Chew, and P. Roy, A front-tracking lattice Boltzmann method to study flowinduced deformation of three-dimensional capsules, Comput. Fluids, 39 (2010), pp. 499-511.

[27] A. Yazdani and P. Bagchi, Influence of membrane viscosity on capsule dynamics in shear flow, J. Fluid Mech., 718 (2013), pp. 569-595.

[28] A. Z. Yazdani and P. Bagchi, Phase diagram and breathing dynamics of a single red blood cell and a biconcave capsule in dilute shear flow, Phys. Rev. E, 84 (2011), p. 026314.

[29] J. Zhang, P. C. Johnson, and A. S. Popel, An immersed boundary lattice Boltzmann approach to simulate deformable liquid capsules and its application to microscopic blood flows, Phys. Biol., 4 (2007), p. 285. 